\title{
Active Vibration Control of a Fluid-Conveying Functionally Graded Cylindrical Shell using Piezoelectric Material
}

\author{
Dan Wang ${ }^{1, *}$, Changqing Bai $^{1}$, and Hongyan Zhang ${ }^{2}$ \\ ${ }^{1}$ State Key Laboratory for Strength and Vibration of Mechanical Structures / Shaanxi Key Laboratory of Environment and Control for \\ Flight Vehicle, Xi'an Jiaotong University, Xi'an 710049, China \\ ${ }^{2}$ School of Science, Chang'an University, Xi'an 710064, China
}

\begin{abstract}
Active vibration control of a smart FG (functionally graded) cylindrical shell conveying fluid in thermal environment is studied theoretically by using a laminated piezoelectric actuator. Velocity feedback control law is implemented to activate the piezoelectric actuator. Considering the electric-thermo-fluidstructure interaction effect, a nonlinear dynamic model of the smart fluid-conveying FG cylindrical shell is developed based on Hamilton's principle and von-Karman type geometrical nonlinear relationship. The inviscid, incompressible, isentropic and irrotational fluid is coupled into governing equations using the linearized potential theory. The Galerkin's method is used to obtain the nonlinear governing equations of motion of the coupled system. The multiple time scales approach is applied to solve the resulting governing equations for analysing the nonlinear dynamic characteristics of the coupled system. The influence of fluid flow velocity, feedback control gains of piezoelectric voltage, external excitation and material properties of FGM on the frequency-response curves of system are investigated. The results indicate that the piezoelectric voltage is an effective controlling parameter for vibration control of the system, and the flow velocity can effect significantly the vibration amplitude and nonlinearity of the coupled system.
\end{abstract}

\section{Introduction}

Cylindrical shells conveying fluid have widely applications in various engineering fields, particularly in aerospace, marine industry, biomechanical applications. These structures are prone to undergoing undesirable vibration and noise due to the coupled effect of fluidstructure interactions, which not only degrade the system performance but also influence the structural integrity and reliability. In order to ensure the safety of the systems and obtain accurate control, the piezoelectric materials are applied in fluid-conveying cylindrical shells to control the coupled vibration.

Functionally graded materials (FGM) have received considerable attention in engineering communities due to the advantages of being able to withstand severe hightemperature while maintaining structural integrity. Piezoelectric FGM structures will have the advantages of FGMs and piezoelectric materials linked together.

A number of works have been employed to study the active control of nonlinear vibration of single structures such as beams, plates and shells without considering fluid effects. Li et al. [1] investigated the active control of a beam subjected to a harmonic excitation using piezoelectric material. Vedat [2] studied the active control of the nonlinear vibration of the FGM plate under random excitation. By using numerical simulations and experimental method, Zhang et al. [3] studied the active vibration control of a cylindrical shell with a laminated PVDF actuator. Considering fluid effects, some attention has been paid to explore the active control of coupled vibration due to fluid-structure interaction by numerical, experimental method and finite element method. Shigeki et al. [4] employed a numerical method to study the active control by using piezoelectric materials for fluid-structure interaction problems. Woo et al. [5] experimentally investigated the active vibration control performance and modal characteristics of a cylindrical shell in air and water. Ray and Reddy [6] analysed the active control performance of a fluidconveying cylindrical shell by using finite element method. In current study, Considering the electricthermo-fluid-structure interaction effect, the nonlinear vibration of a smart FG cylindrical shell conveying fluid in thermal environment is studied theoretically. And the active vibration control of the coupled system is investigated by using a laminated piezoelectric actuator.

\section{Model description and nonlinear dynamic modeling}

A smart FG cylindrical shell conveying fluid with length $L$, thickness $h$ and mean radius $R$ is considered herein, as shown in Fig.1. The piezoelectric actuator layer with thickness $h_{p}$ is perfectly bonded onto the outer surface of FG cylindrical shell, which is used to control the coupled vibration of fluid-conveying FG shell. It is polarized along the thickness direction. The fluid with density, $\rho_{f}$,

\footnotetext{
* Corresponding author: wd8625701371@163.com
} 
flows in the $x$-direction of the cylindrical shell with a uniform flow velocity $U_{f}$. The FGM shell is subjected to a radial harmonic excitation $q(x, \theta, t)$ with excitation amplitude $q_{0}$ and excitation frequency $\Omega$. The system is defined in a coordinate system $(x, \theta, z)$ as shown in Fig.1, where $x, \theta$ and $z$ are the axial, circumferential and radial coordinates, respectively. The corresponding displacement components are denoted by $u, v$ and $w$, respectively.

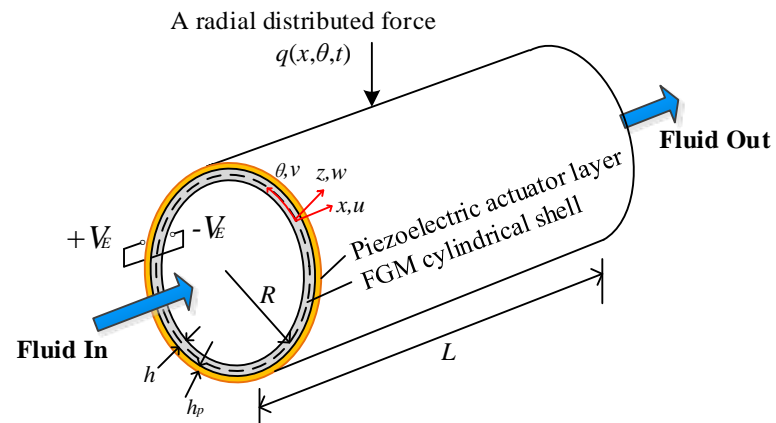

Fig. 1. Schematic model of a smart FG cylindrical shell conveying fluid with a laminated piezo-actuator.

\subsection{Theoretical formulations}

The FGM cylindrical shell is composed of ceramics and metals. In thermal environment, the effective material properties of FGM cylindrical shell, $P_{\text {eff, }}$, are related to both the thickness of shell and temperature, which can be expressed as

$$
P_{\text {eff }}(z, T)=\left[P_{m}(T)-P_{c}(T)\right]\left(\frac{z+h / 2}{h}\right)^{\eta}+P_{c}(T)
$$

where $\eta$ expresses the volume fraction exponent. $P_{m}(T)$ and $P_{c}(T)$ denote the properties of metal and ceramic of FGM shell, respectively. It can be defined as a function of temperature as

$$
P(T)=P_{0}\left(P_{-1} T^{-1}+1+P_{1} T+P_{2} T^{2}+P_{3} T^{3}\right)
$$

where $P_{0}, P_{-1}, P_{1}, P_{2}, P_{3}$ are the temperature coefficients, which are unique to constituent materials. The temperature distribution of FGM cylindrical shell is assumed to vary linearly along the thickness.

The FGM cylindrical shell is modeled based on Donnell shell's theory with taking von Karman geometrical nonlinearity into account. The straindisplacement relations can be written as

$$
\left\{\begin{array}{l}
\varepsilon_{x x} \\
\varepsilon_{\theta \theta} \\
\varepsilon_{x \theta}
\end{array}\right\}=\left\{\begin{array}{l}
\frac{\partial u}{\partial x}+\frac{1}{2}\left(\frac{\partial w}{\partial x}\right)^{2} \\
\frac{1}{R} \frac{\partial v}{\partial \theta}+\frac{w}{R}+\frac{1}{2 R^{2}}\left(\frac{\partial w}{\partial \theta}\right)^{2} \\
\frac{1}{R} \frac{\partial u}{\partial \theta}+\frac{\partial v}{\partial x}+\frac{1}{R} \frac{\partial w}{\partial \theta} \frac{\partial w}{\partial x}
\end{array}\right\}+z\left\{\begin{array}{l}
-\frac{\partial^{2} w}{\partial x^{2}} \\
-\frac{1}{R^{2}} \frac{\partial^{2} w}{\partial \theta^{2}} \\
-\frac{1}{R} \frac{2 \partial^{2} w}{\partial x \partial \theta}
\end{array}\right\}
$$

The stress-strain relations of the FGM cylindrical shell considering temperature effects, are

$$
\left\{\begin{array}{c}
\sigma_{x x} \\
\sigma_{\theta \theta} \\
\tau_{x \theta}
\end{array}\right\}=\left[\begin{array}{ccc}
Q_{11} & Q_{12} & 0 \\
Q_{21} & Q_{22} & 0 \\
0 & 0 & Q_{66}
\end{array}\right]\left\{\begin{array}{c}
\varepsilon_{x x}-\alpha_{11} \Delta T_{1} \\
\varepsilon_{\theta \theta}-\alpha_{22} \Delta T_{1} \\
\varepsilon_{x \theta}
\end{array}\right\}
$$

where the thermal expansion coefficients $\alpha_{11}=\alpha_{22}=\alpha_{\text {eff }}$, and $\Delta T_{1}=T(z)-T_{m}$. The elasticity coefficients are defined as $Q_{11}=Q_{22}=E_{\text {eff }} /\left(1-v_{\text {eff }}^{2}\right)$, $Q_{12}=Q_{21}=E_{\text {eff }} v_{\text {eff }} /\left(1-v_{\text {eff }}^{2}\right), Q_{66}=E_{\text {eff }} /\left[2\left(1+v_{\text {eff }}\right)\right]$. The effective mass density $\rho_{\text {eff }}$, the effective Young's modulus $E_{\text {eff, }}$, the effective Poisson's ratio $v_{\text {eff }}$ and the thermal conductivity $\alpha_{\text {eff }}$ vary according to Eqs. (1-2).

For the piezoelectric actuator layer, the constitutive equations coupling elastic, thermal and electric fields in the piezoelectric medium, can be expressed as

$$
\begin{aligned}
& \left\{\begin{array}{l}
\sigma_{x x}^{p} \\
\sigma_{\theta \theta}^{p} \\
\tau_{x \theta}^{P}
\end{array}\right\}=\left[\begin{array}{ccc}
C_{11} C_{12} & 0 \\
C_{12} & C_{22} & 0 \\
0 & 0 & C_{66}
\end{array}\right]\left\{\begin{array}{c}
\varepsilon_{x x}-\alpha_{11 e} \Delta T_{2} \\
\varepsilon_{\theta \theta}-\alpha_{22 e} \Delta T_{2} \\
\varepsilon_{x \theta}
\end{array}\right\}-\left[\begin{array}{lll}
0 & 0 & e_{31} \\
0 & 0 & e_{32} \\
0 & 0 & 0
\end{array}\right]\left\{\begin{array}{l}
E_{x} \\
E_{\theta} \\
E_{z}
\end{array}\right\} \\
& D_{z}=e_{31} \varepsilon_{x x}+e_{32} \varepsilon_{\theta \theta}+\in_{33} E_{z}
\end{aligned}
$$

where the piezoelectric actuator layer is subjected to a temperature variation $\Delta T_{2}$, and $\Delta T_{2}=T_{p}(z)-T_{0}\left(T_{0}=\right.$ $300 \mathrm{~K})$. The temperature distribution of piezoelectric layer, $T_{p}(z)$, varies linearly along the thickness. $E_{i}$ and $D_{i}$ are the electric field intensities and electric displacements. $C_{i j}, e_{i j}$ and $\in_{i j}$ represent the elastic, piezoelectric and dielectric constants, respectively. $\alpha_{11 e}$ and $\alpha_{22 e}$ are the thermal expansion coefficients of piezoelectric layer in the $x$ and $\theta$ directions.

A layerwise quadratic distribution of the electric potential $\varphi$ is considered and given by

$$
\varphi(x, \theta, z, t)=\frac{2 z_{p}}{h_{p}} V_{E}+\left[z_{p}{ }^{2}-\left(\frac{h_{p}}{2}\right)^{2}\right] \psi(x, \theta, t)
$$

where $z_{p}$ is the local thickness coordinate with respect to the piezoelectric layer mid-plane, $z_{p}=z-\left(h+h_{p}\right) / 2 . V_{E}$ is the electric voltage applied to the piezoelectric layer. $\psi$ is the induced electric potential by elastic deformation in the piezoelectric element. The electric field intensities $E_{z}=-\varphi_{, z}$.

\subsection{Equations of motion of the smart FG cylindrical shell conveying fluid}

The Hamilton's principle is utilized to develop the governing equations of motion of the smart FG cylindrical shell conveying fluid subjected to an external radial harmonic excitation.

$$
\int_{t_{1}}^{t_{2}}\left(\delta T-\delta U+\delta W_{f}+\delta W_{e x c}\right) d t=0
$$

where $T$ and $U$ are referred to the total kinetic energy and potential energy of the system. $W_{\text {exc }}$ is the work done by the external excitation. $W_{f}$ is the work done by fluid perturation pressure. 


$$
\begin{gathered}
U=\frac{1}{2} \iiint_{V_{s}}\left(\begin{array}{l}
\sigma_{x x}\left(\varepsilon_{x x}-\alpha_{11} \Delta T_{1}\right) \\
+\sigma_{\theta \theta}\left(\varepsilon_{\theta \theta}-\alpha_{22} \Delta T_{1}\right)+\tau_{x \theta} \varepsilon_{x \theta}
\end{array}\right) d V_{s} \\
+\frac{1}{2} \iiint_{V_{p}}\left[\begin{array}{l}
\sigma_{x x}^{p}\left(\varepsilon_{x x}-\alpha_{11}^{e} \Delta T_{2}\right) \\
+\sigma_{\theta \theta}^{p}\left(\varepsilon_{\theta \theta}-\alpha_{22}^{e} \Delta T_{2}\right) \\
+\tau_{x \theta}^{p} \varepsilon_{x \theta}-D_{z} E_{z}
\end{array}\right] d V_{p} \\
T=\frac{1}{2} \iiint_{V_{s}}\left[\rho_{1}(z)\left(\dot{u}^{2}+\dot{v}^{2}+\dot{w}^{2}\right)\right] d V_{s} \\
+\frac{1}{2} \iiint_{V_{p}}\left[\rho_{2}\left(\dot{u}^{2}+\dot{v}^{2}+\dot{w}^{2}\right)\right] d V_{p} \\
W_{e x c}=\int_{0}^{2 \pi} \int_{0}^{L} q(t) w d x R d \theta
\end{gathered}
$$

The fluid in the FG cylindrical shell is considered to be inviscid, incompressible, isentropic and irrotational. The equations of the smart piezoelectric FG cylindrical shell - fluid system are coupled via the work done by fluid perturbation pressure which can be written as

$$
W_{f}=\int_{0}^{2 \pi} \int_{0}^{L} P_{p} w d x R d \theta
$$

The fluid perturbation pressure is related to shell motions and act on the radial direction of the FGM cylindrical shell. The fluid-structure interaction can be described by the linear potential flow theory. The velocity potential, $\Psi$, can be defined as the sum of the two parts including a steady part due to the mean flow velocity $U_{f}$ in the $x$ direction and an unsteady part $\Phi$ related to shell motion, $\Psi=U_{f} x+\Phi$. $\Phi$ satisfies the Laplace equation $\nabla^{2} \Phi=\Phi_{, r r}+\frac{1}{r} \Phi_{, r}+\frac{1}{r^{2}} \Phi_{, \theta \theta}+\Phi_{, x x}=0$, no cavitation occurs at the shell-fluid interface, $\left.\Phi_{, r}\right|_{r=R}=\left(w_{, t}+U_{f} w_{, x}\right) \quad$. Based on the unsteady Bernoulli's equation, the perturbation pressure $p$ is obtained, $p=-\rho_{f}\left(\Phi_{, t}+U_{f} \Phi_{, x}\right)$, which can be expressed as

$$
P_{p}=-\rho_{f} \sum_{m=1}^{M} \sum_{n=0}^{N} \frac{L}{m \pi} \frac{I_{n}(m \pi R / L)}{I_{n}^{\prime}(m \pi R / L)}\left(\frac{\partial}{\partial t}+U_{f} \frac{\partial}{\partial x}\right)^{2} w(12
$$

where $\boldsymbol{I}_{n}$ is modified Bessel function of the first kind of order $n . I_{n}^{\prime}$ is its first derivate.

Substituting Eqs. (8-12) into Eq. (7) and rearranging the governing equations, yields the nonlinear coupled differential equations as follows

$$
\begin{aligned}
& L_{11}(u)+L_{12}(v)+L_{13}(w)+L_{14}(\psi)+\hat{L}_{1}(w)=I_{11} \frac{\partial^{2} u}{\partial t^{2}} \\
& L_{21}(u)+L_{22}(v)+L_{23}(w)+L_{24}(\psi)+\hat{L}_{2}(w)=I_{11} \frac{\partial^{2} v}{\partial t^{2}} \\
& L_{31}(u)+L_{32}(v)+L_{33}(w)+L_{34}(\psi)+\hat{L}_{3}(w)+Q_{3}(u, w) \\
& +R_{3}(v, w)+\frac{N_{\theta}^{T}+N_{\theta p}^{E}}{R}+q(x, \theta, t)+P_{p}=I_{11} \frac{\partial^{2} w}{\partial t^{2}}+c_{w} \frac{\partial w}{\partial t} \\
& L_{41}(u)+L_{42}(v)+L_{43}(w)+L_{44}(\psi)+\hat{L}_{4}(w)=J_{E}
\end{aligned}
$$

\section{Active control of nonlinear vibration for the coupled system}

An analytical approach is utilized to investigate the nonlinear vibrations of the coupled system. The simplysupported boundary condition is considered at two ends of the cylindrical shell. The shell displacements are discretized by using the following trigonometric expansions.

$$
\begin{aligned}
& u(x, \theta, t)=\sum_{m=1}^{M} \sum_{n=0}^{N} U_{m n}(t) \cos \left(\lambda_{m} x\right) \cos (n \theta) \\
& v(x, \theta, t)=\sum_{m=1}^{M} \sum_{n=1}^{N} V_{m n}(t) \sin \left(\lambda_{m} x\right) \sin (n \theta) \\
& w(x, \theta, t)=\sum_{m=1}^{M} \sum_{n=0}^{N} W_{m n}(t) \sin \left(\lambda_{m} x\right) \cos (n \theta) \\
& \psi(x, \theta, t)=\sum_{m=1}^{M} \sum_{n=0}^{N} \psi_{m n}(t) \sin \left(\lambda_{m} x\right) \cos (n \theta)
\end{aligned}
$$

where $\lambda_{m}=m \pi / L, U_{m n}, V_{m n}, W_{m n}, \psi_{m n}$ are the timedependent generalized coordinates. $m$ and $n$ are axial and circumferential wavenumbers. Substituting Eq.(14) into Eq.(13) and then employing Galenkin method, the nonlinear ordinary differential equation can be obtained. By omtting the in-plane inertias, then the transverse motion of the coupled system can be obtained.

$$
\begin{aligned}
& \ddot{W}_{m n}+a_{1} \dot{W}_{m n}+a_{2} W_{m n}+a_{3} W_{i j} W_{p q} \\
& +a_{4} W_{i j} W_{p q} W_{r s}=q_{0} \cos (\Omega t)+f_{p} \cdot V_{E}
\end{aligned}
$$

where $f\left(V_{E}\right)$ is the control force of piezoelectric actuator, and $f_{p} \cdot V_{E}=a_{5} V_{E}+a_{6} W_{m n} V_{E}$. The control voltage $V_{E}$ supplied to the piezoelectric actuator layer is considered to be negatively proportional to the velocity of a point on the outer surface of the shell, which can be expressed as

$$
V_{E}=-G_{v} \dot{w}\left(x_{p}, \theta_{p}, t\right)=-G_{v} C \dot{W}_{m n}
$$

where $G_{v}$ is the control gain, and $C$ is the transform vector used to transfer the coordinates of the feedback measurement point $\left(x_{p}, \theta_{p}\right)$ from modal coordinates to cylindrical coordinates.

Substituting Eq.(16) into Eq.(15), the transverse motion of the coupled system can be rewritten as

$$
\begin{aligned}
& \ddot{W}_{m n}+\left(a_{1}+f_{p} G_{v} C\right) \dot{W}_{m n}+a_{2} W_{m n} \\
& +a_{3} W_{i j} W_{p q}+a_{4} W_{i j} W_{p q} W_{r s}=q_{0} \cos (\Omega t)
\end{aligned}
$$

Multiple time scales method is utilized to obtain the approximate analytical solution of Eq. (17). The Eq. (17) can be rewritten in the following form

$$
\begin{aligned}
& \ddot{W}_{m n}+\omega_{m n}^{2} W_{m n}+\varepsilon^{2} \alpha_{1} \dot{W}_{m n}+\varepsilon^{2} \alpha_{2} W_{m n} \dot{W}_{m n} \\
& +\varepsilon \alpha_{3} W_{m n}^{2}+\varepsilon^{2} \alpha_{4} W_{m n}^{3}=\varepsilon^{2} \kappa_{m n} \cos (\Omega t)
\end{aligned}
$$

in which

$$
\begin{aligned}
& \omega_{m n}^{2}=a_{2}, \alpha_{1}=\left(a_{1}+a_{5} G_{v} C\right) / \varepsilon^{2}, \alpha_{2}=a_{6} G_{v} C / \varepsilon^{2}, \\
& \alpha_{3}=a_{3} / \varepsilon, \alpha_{4}=a_{4} / \varepsilon^{2}, \kappa_{m n}=q_{0} / \varepsilon^{2}
\end{aligned}
$$

The primary resonace is considered in the present study. The detuning parameter $\sigma$ is introduced in order to express the nearness of the excitation frequency $\Omega$ to the eigenfrequency $\omega_{m n}, \Omega=\omega_{m n}+\varepsilon^{2} \sigma$. The frequency- 
amplitude curves of the smart fluid-conveying FG cylindrical shell bonded with a laminated piezoelectric actuator, is then deduced for the desire mode.

$$
\frac{\Omega}{\omega_{m n}}=1+\frac{\varepsilon^{2} A^{2}}{8 \omega_{m n}^{2}}\left[3 \alpha_{4}-\frac{10 \alpha_{3}^{2}}{3 \omega_{m n}^{2}}\right] \pm \frac{\varepsilon^{2}}{\omega_{m n}} \sqrt{\frac{\kappa_{m n}^{2}}{4 \omega_{m n}^{2} A^{2}}-\alpha_{1}^{2}}
$$

\section{Results and discussions}

In order to verify the validity of the present method, the natural frequencies of a homogeneous piezoelectric made of PZT-4 without conveying fluid are compared with the existing analytical results [7]. The comparison results are shown in Table 1 , and good agreements can be observed.

Table 1. Natural frequencies $(\mathrm{GHz})$ of piezoelectric cylindrical shells with $m=1$.

\begin{tabular}{|c|c|c|}
\hline$n$ & Ke et. al. [7] & Present \\
\hline 1 & 0.4583 & 0.4563 \\
\hline 2 & 0.2433 & 0.2458 \\
\hline 3 & 0.5301 & 0.5298 \\
\hline 4 & 1.0039 & 1.1001 \\
\hline 5 & 1.6199 & 1.5998 \\
\hline 6 & 2.3738 & 2.3701 \\
\hline 7 & 3.2655 & 3.2679 \\
\hline 8 & 4.2944 & 4.3002 \\
\hline 9 & 5.4606 & 5.4605 \\
\hline 10 & 6.7640 & 6.7832 \\
\hline
\end{tabular}

The effect of various parameters on the frequencyresponse curves of primary resonance for the smart fluidconveying FG cylindrical shell bonded with a laminated piezoelectric actuator and subjected to the harmonic external excitation are shown in Fig.2. The FGM cylindrical shell layer is $\mathrm{ZrO}_{2}$ (ceramic material)/ $\mathrm{T}_{\mathrm{i}}-6 \mathrm{Al}-$ $4 \mathrm{~V}$ (metal material). The outer piezoelectric layer is PZT4. The parameters used are $L=0.3, R=0.6, h=0.003$, $h_{p}=h / 3, \eta=2$. The feedback measurement point is selected as $\left(x_{p}, \theta_{p}\right)=(L / 2,0)$.

The effect of the external excitation amplitude on amplitude-frequency response of the system is plotted in Fig.2(a). It can be seen that the resonant region and the system nonlinearity increase with the increasing excitation amplitude, and the vibration amplitude increases as the excitation amplitude increases. Fig.2(b) shows the effect of the feedback control gains of the piezoelectric voltages on amplitude-frequency response of the system. It can be observed that vibration amplitudes decrease with the increasing feedback control gains. This indicates the larger control gains applied to the piezoelectric actuator can control the system vibtation. Fig.2(c) shows the effect of fluid velocity on the amplitude-frequency response of the system. It can be seen the vibration amplitude increases as the flow velocity increase. The reason of this change can be explained by the fact that the fluid perturbation pressure $P_{p}$ at the shell wall increases as the flow velocity increases. This leads to a decrease in the system stiffness and increase the vibration amplitude. Fig.2(d) describes the effect of material property of the FGM cylindrical shell on the amplitude-frequency response of the system. As the volume fraction exponent increases, the resonance amplitudes decrease and the hardening nonlinearity of the coupled system increases. This is due to the ceramic content ( $\mathrm{ZrO} 2)$ in functionally graded material increases as the volume fraction exponent increases, and the elastic modulus of ceramic ( $\mathrm{ZrO} 2)$ is larger than that of their metal counterpart (Ti-6Al-4V).

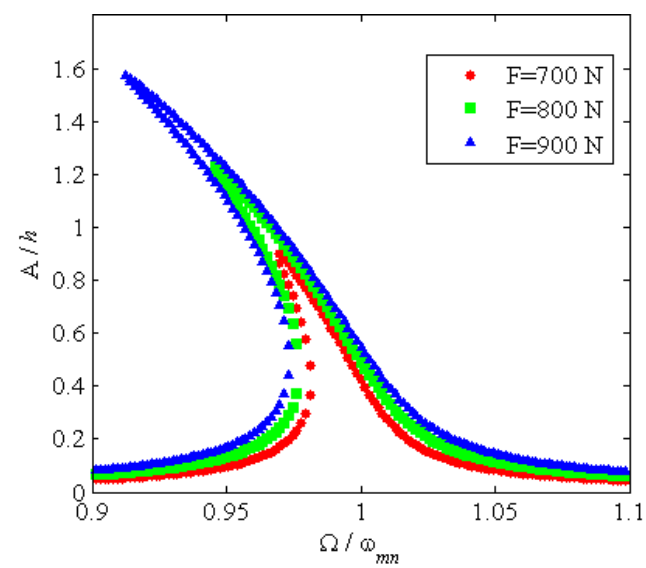

(a)

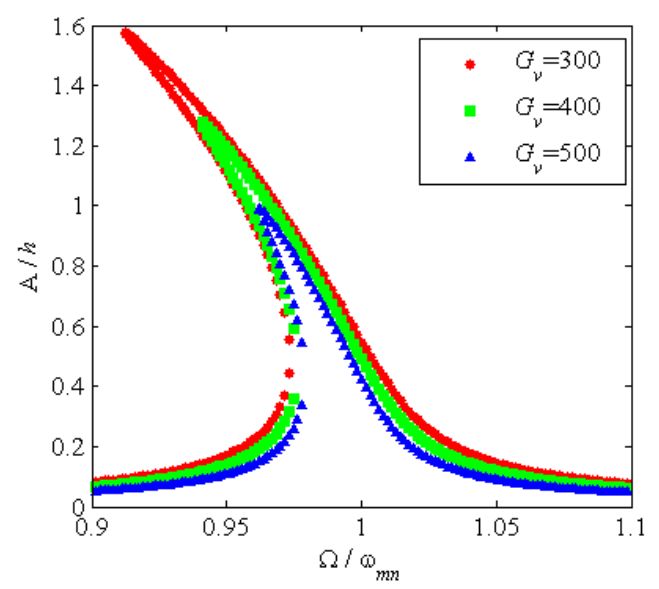

(b)

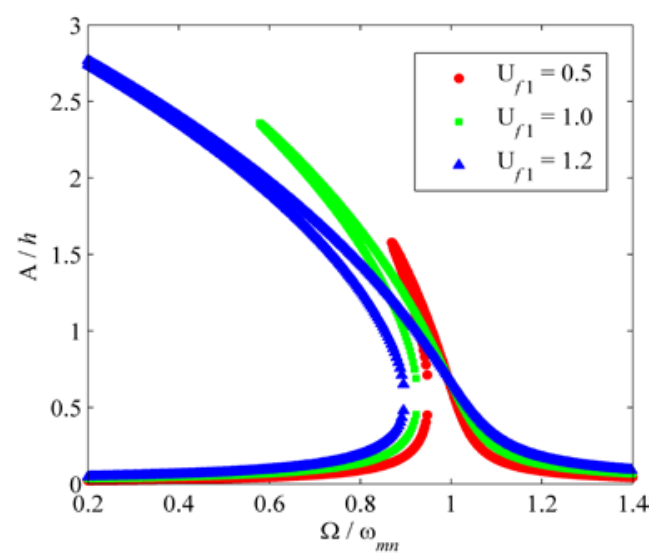

(c) 


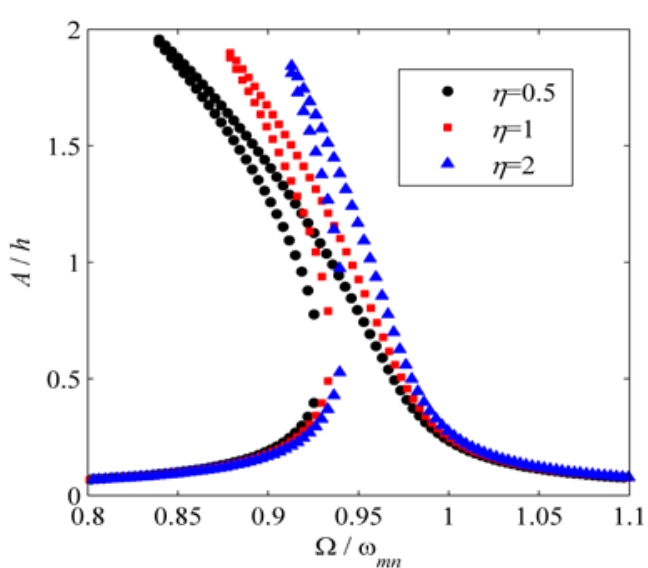

(d)

Fig. 2. Effect of various parameters on the frequency-response curves of the smart fluid-conveying FG cylindrical shell: (a) external excitation amplitude, (b) feedback control gains, (c) flow velocity, (d) volume friction of FGM.

\section{Conclusions}

Active vibration control of a smart fluid-conveying FG cylindrical shell bonded with a laminated piezoelectric actuator in thermal environment is studied theoretically. Velocity feedback control law is implemented to activate the piezoelectric actuator. Considering electric-thermofluid-structure interaction effect, a nonlinear dynamic model of system is developed. The effects of various parameters on nonlinear dynamic behaviours of the system are discussed. The results indicate that the piezoelectric voltage is an effective controlling parameter for vibration control of the system, and the fluid flow velocity can effect significantly the vibration amplitude and the nonlinearity of the coupled system. The theoretical framework and numerical results presented in this paper are helpful for the application of smart structures subjected to internal fluid flow under harmonic excitation.

The authors would like to acknowledge the support of the Fundamental Research Funds for the Central Universities (Grant No. xjj2018220).

\section{References}

1. Fengming Li, Guo Yao, and Yimin Zhang. Active control of nonlinear forced vibration in a flexible beam using piezoelectric material. Mech. Adv. Mater. Struc., 23(3): 311-17 (2016).

2. Dogan Vedat. Active vibration control of functionally graded plates under random excitation. J. Intel. Mat. Syst. Str., 26(11): 1370-84 (2015).

3. Yahong Zhang, Hongpan Niu, Shilin Xie and Xinong Zhang. Numerical and experimental investigation of active vibration control in a cylindrical shell partially covered by a laminated PVDF actuator. Smart Mater. Struct., 17(3) (2008).

4. Kaneko Shigeki, Giwon Hong, Naoto Mitsume, Tomonori Yamada, and Shinobu Yoshimura. Numerical study of active control by piezoelectric materials for fluid-structure interaction problems. J. Sound Vib., 435: 23-35 (2018).

5. Sohn Jung Woo, Seung-Bok Choi. Active vibration control of a cylindrical structure using flexible piezoactuators: experimental work in air and water environments. Smart Mater. Struct., 23(11): 1-9 (2014).

6. Ray MC, Reddy JN. Active damping of laminated cylindrical shells conveying fluid using 1-3 piezoelectric composites. Compos. Struct., 98: 26171 (2013).

7. LL Ke, YS Wang, Reddy JN. Thermo-electromechanical vibration of size-dependent piezoelectric cylindrical nanoshells under various boundary conditions. Compos. Struct., 116: 626-36 (2014). 